\title{
APROXIMACIÓN AL ESTUDIO DE LA EVALUACIÓN EN LA UNIVERSIDAD: UN ANÁLISIS EXPLORATORIO CON ALUMNOS SEMIPRESENCIALES
}

\author{
Ana Gargallo Castel \\ Universidad de Zaragoza
}

\begin{abstract}
RESUMEN: En este trabajo se revisa la importancia de la evaluación como instrumento de mejora dentro del proceso de enseñanza-aprendizaje y la necesidad de coherencia con el resto de elementos del proceso. Para ello se realizó una encuesta a un grupo de alumnos semipresenciales sobre las metodologías y los diferentes modelos evaluativos, el método evaluativo que consideran más interesante, y en general el cómo, el cuándo y el dónde. Los resultados ofrecen un análisis exploratorio sobre las preferencias de los alumnos en relación con su evaluación y reflejan algunas diferencias con respecto a los modelos evaluativos actuales.
\end{abstract}

ABSTRACT: This study reviews the importance of the evaluation to improve the teaching - learning process and the importance of the coherence with the rest of elements of the process. A survey about methodologies, models of evaluation and preferences was realized to a group of semipresencial students. The results offer an exploratory analysis on the preferences of the students and reflect some differences with regard to the current evaluation models.

PALABRAS CLAVE: Proceso de educación, enseñanza-aprendizaje, evaluación del estudiante, evaluación formativa, evaluación sumativa, actividades de aprendizaje.

KEYWORDS: Process education, teaching-learning process, student evaluation, formative evaluation, summative evaluation, learning activities.

\section{INTRODUCCIÓN}

El mundo de la enseñanza se encuentra en una situación de necesaria evolución y reflexión para adaptarse a las nuevas demandas educativas y sociales. Para ello, debemos situarnos en un paradigma de enseñanza centrada en el aprendizaje para lo cual debe conseguirse una evaluación para el aprendizaje y no en una evalua- 
ción del aprendizaje (Escudero, 2010). A pesar de la evolución en la concepción de la evaluación educativa, a la evaluación se le ha seguido atribuyendo en muchos casos un papel independiente del resto del proceso de enseñanza, sin ningún tipo de consecuencia sobre el mismo. Sin embargo en la actualidad se acepta que la evaluación está íntimamente ligada al proceso de aprendizaje, siendo numerosos los estudios en la literatura especializada que destacan el impacto de la evaluación en la calidad del aprendizaje (Bordas y Cabrera, 2001). Las características de la evaluación utilizada por los profesores para valorar el aprendizaje de los alumnos, va a incidir en la motivación y en el comportamiento de los estudiantes, siendo un factor clave, en mayor medida incluso que los objetivos del curriculum y los métodos de enseñanza (Biggs, 1996). Puede decirse que la evaluación es uno de los elementos que mayor impacto ejerce en la conducta del estudiante en el proceso de aprendizaje (Campanario, 1998), por lo que resulta clara su importancia como recurso para favorecer el aprendizaje de los alumnos y para reflexionar sobre la enseñanza en su conjunto (Rosales, 2000). Por tanto, si se desea modificar los procedimientos de aprendizaje de los alumnos, debería modificarse entre otras cosas la evaluación (Pérez et al., 2000).

Además no debe olvidarse que en las instituciones universitarias la evaluación constituye uno de los elementos principales de su funcionamiento al ofrecer información para valorar la calidad de los procesos educativos y de los resultados de los mismos (Pinelo, 2005). En este trabajo se desea profundizar en la opinión de los estudiantes sobre la evaluación de su aprendizaje en la universidad, en concreto en la modalidad de enseñanza semipresencial en la Facultad de Ciencias Sociales y Humanas de la Universidad de Zaragoza, localizada en el campus de Teruel.

\section{Distintas eValuaciones}

De acuerdo con la perspectiva constructivista, la evaluación debe considerarse como un elemento impulsor del aprendizaje y del trabajo del alumno, como una oportunidad de aprendizaje (Crooks, 1988). Si bien durante muchos años el objetivo de la educación fue la formación de estudiantes con conocimiento en un dominio, se está produciendo un giro hacia la adquisición de destrezas de alto nivel, la resolución de problemas bajo presión o un aprendizaje manifiesto como por ejemplo el aprendizaje en contextos de vida real (Dochy, 2001) por lo que no debería ser un ejercicio rutinario ni de memorización (Campanario, 1998). Además, debe existir coherencia entre las metas educativas que se proponen en la asignatura y los criterios evaluativos aplicados. En muchas ocasiones las prácticas de evaluación no son consistentes con las metas de aprendizaje propuestas, se proponen objetivos relacionados con el desarrollo del pensamiento crítico mientras que se aplican criterios de evaluación que favorecen el aprendizaje repetitivo frente a la aplicación de destrezas de alto nivel (Bol y Strage, 1996).

En todo caso, en muchas ocasiones no se le otorga a las prácticas evaluativas la importancia que se merece, asignándole un papel meramente regulador y de control, sin conocer ni aprovechar las potencialidades dados los efectos que presentan en los estudiantes en el corto, mediano y largo plazo (Córdoba, 2006). 
En los próximos apartados se recogen algunas de las tipologías más utilizadas por los expertos para diferenciar tipos de evaluación dependiendo de las funciones que desempeña, de los criterios utilizados para la evaluación, etc.

\subsection{Tipos de evaluación según su funcionalidad}

Atendiendo a la funcionalidad que se persigue mediante la evaluación, la clasificación más utilizada recoge tres categorías de evaluación:

\section{Evaluación inicial}

La Evaluación Inicial o Predictiva (Diagnóstica) tiene por objeto proporcionar a los docentes información sobre los conocimientos previos de los alumnos (Rosales, 1981), a la vez que ayuda a éstos a ser conscientes de ellas. Tiene carácter diagnóstico y se realiza al inicio de cada ciclo didáctico: unidades didácticas, secuencias o tareas específicas definidas para alcanzar una competencia concreta.

Permite mejorar la organización didáctica, implementar los ajustes que sean precisos en los objetivos, contenidos y criterios en función de las características del grupo o del individuo. Con ello se consigue diseñar estrategias de aprendizaje que permitan construir la enseñanza a partir de las ideas y capacidades previas de los alumnos.

En ocasiones se considera que esta evaluación viene implícitamente marcada por las competencias que comprende el curso o período anterior (Castro et al., 2004), sin embargo esta suposición no siempre es tan clara, por lo que resulta interesante efectuar este tipo de evaluación.

Esta puede llevarse a cabo a través de recursos escritos tales como los tests de elección múltiple o las cuestiones abiertas o bien por medios orales como puede ser el realizar preguntas individualizadas al inicio de cada unidad didáctica en las sesiones presenciales de las que disponen los alumnos semipresenciales.

Por último, los resultados de la evaluación diagnóstica o inicial pueden comentarse brevemente inmediatamente después de su realización o bien se pueden abordar a lo largo del curso coincidiendo con el momento del desarrollo de los contenidos correspondientes.

\section{Evaluación estímulo}

La evaluación estímulo, también conocida como evaluación de proceso, evaluación intermedia o evaluación formativa, es aquella que se realiza durante el proceso de enseñanza-aprendizaje, tendente a calificar aquel proceso de evaluación al servicio de un programa en desarrollo, con objeto de mejorarlo (Scriven, 1967). Recoge la retro-información periódica centrada en el conocimiento de los resultados de evaluaciones relajadas cortas y rápidas, con escasos requisitos métricos, ajustadas a las unidades temáticas (Escudero, 2010). Está asociada a la utilidad de la evaluación como instrumento de mejora del proceso, ofreciendo información sobre qué aprenden los estudiantes y cómo lo aprenden. Con ella se consigue ofrecer una retroalimentación inmediata al alumno de los logros obtenidos, y en su caso, de donde y en que nivel existen dificultades de aprendizaje. Del mismo modo, ofrece 
a los profesores elementos para reajustar sus métodos y estrategias pedagógicas, con el objetivo de mejorar el proceso de aprendizaje sin necesidad de llegar al final del mismo para descubrir los posibles problemas.

Esta característica de la evaluación formativa es la que ha hecho pensar a algunos pedagogos que se trata de la modalidad evaluativa más acorde con el modelo curricular (Castro et al., 2004). Las ventajas de esta evaluación hacen referencia a las cuestiones siguientes (Escudero, 2010):

1. Proporciona información sobre puntos fuertes y débiles en el aprendizaje, ayudando a los alumnos a progresar en su aprendizaje.

2. Facilita a los alumnos el progreso en su aprendizaje, actuando como orientación y apoyo en esa tarea, constituyendo una fuente de motivación extrínseca.

3. Ayuda a los profesores a evaluar su propia efectividad obligándoles a estructurar y contextualizar el proceso.

4. Refuerza la actividad de los alumnos.

La evaluación estímulo puede llevarse a cabo a través de la revisión de actividades por parte del propio alumno individualmente (autoevaluación, autoaprendizaje y autocorrección) o bajo la forma de coevaluación o evaluación por parejas bien en las sesiones presenciales o en los foros habilitados para ello.

Además el profesor puede realizar los conocidos exámenes parciales con los que el alumno además se familiariza con el formato de cara al examen final. A continuación conviene realizar una corrección y discusión de los resultados en clase para que la efectividad de la evaluación sea mayor (Bangert-Drowns et al., 1991).

Sin embargo, el profesor puede utilizar la evaluación formativa sin necesidad de realizar exámenes, sino a través de la interacción con los alumnos, su participación en las clases presenciales, en las discusiones mediante los foros correspondientes y en los análisis de grupo presenciales. Uno de los procedimientos más útiles para la evaluación formativa es la utilización de preguntas orales en clase adecuadamente diseñadas para tal fin, o bien las preguntas cortas contestadas por escrito.

\section{Evaluación sumativa}

La evaluación sumativa, también conocida con el nombre de evaluación final o evaluación del producto es aquella que permite comprobar la eficacia del programa y tomar decisiones sobre su continuidad (Scriven, 1967), permite conocer los resultados conseguidos por el alumno al término de una fase de aprendizaje.

El objetivo de este tipo de evaluación es simplemente selectivo o acreditativo, permitiendo otorgar una certificación o acreditación del nivel alcanzado por el individuo tras la finalización de un determinado proceso. En ese marco, el principal y único indicador proviene de los resultados finales en términos de aprobados o suspensos.

La utilización aislada de esta tipología de evaluación impide observar la evolución del proceso, de modo que cualquier problema de programación puede ocasionar grandes perjuicios en la evolución del proceso. 
Para lograr un mayor aprendizaje debe realizarse una evaluación inicial para diagnosticar el punto de partida del alumno y revisar la evolución del proceso de enseñanza-aprendizaje a lo largo del tiempo (Escudero, 1995). Con ello se logra orientar y mejorar el proceso aprovechando las potencialidades de la evaluación inicial, formativa y sumativa. Por tanto puede decirse que la evaluación no es apéndice de la enseñanza y del aprendizaje sino parte importante de los mismos para reforzar, orientar o modificar la planificación si resultase necesario.

Cuadro 1. Resumen de los tipos de evaluación según su funcionalidad.

\begin{tabular}{|c|c|c|c|}
\hline Aspectos & Evaluación inicial & Evaluación estímulo & Evaluación sumativa \\
\hline Función Referencial & $\begin{array}{l}\text { Rol del Diagnóstico: } \\
\text { Determina la } \\
\text { presencia o } \\
\text { ausencia de } \\
\text { objetivos requisitos } \\
\text { de entrada. }\end{array}$ & $\begin{array}{c}\text { Rol Formativo: } \\
\text { Detecta éxitos o } \\
\text { deficiencias para } \\
\text { prescribir } \\
\text { actividades de } \\
\text { profundización. } \\
\text { Retroalimentación. }\end{array}$ & $\begin{array}{l}\text { Rol Sumativo: } \\
\text { Certifica el grado de } \\
\text { logro de los } \\
\text { objetivos con el } \\
\text { propósito de asignar } \\
\text { calificaciones. }\end{array}$ \\
\hline $\begin{array}{l}\text { Momento de su } \\
\text { realización }\end{array}$ & $\begin{array}{l}\text { Al inicio de una } \\
\text { unidad de } \\
\text { aprendizaje. }\end{array}$ & $\begin{array}{c}\text { Durante el proceso } \\
\text { de enseñanza y } \\
\text { aprendizaje. }\end{array}$ & $\begin{array}{l}\text { Al finalizar una o } \\
\text { varias unidades de } \\
\text { aprendizaje. }\end{array}$ \\
\hline $\begin{array}{l}\text { Procedimientos } \\
\text { evaluativos que } \\
\text { utilizan }\end{array}$ & $\begin{array}{l}\text { Procedimiento } \\
\text { prueba y de } \\
\text { observación. }\end{array}$ & $\begin{array}{l}\text { Procedimiento de } \\
\text { prueba y de } \\
\text { observación. }\end{array}$ & $\begin{array}{l}\text { Procedimientos de } \\
\text { prueba, observación } \\
\text { e informe. }\end{array}$ \\
\hline Tipo de objetivo & $\begin{array}{l}\text { Objetivos requisitos } \\
\text { (o conductas de } \\
\text { entrada). }\end{array}$ & $\begin{array}{c}\text { Objetivos } \\
\text { capacitadores, } \\
\text { subordinados o } \\
\text { intermedios. }\end{array}$ & $\begin{array}{l}\text { Objetivos } \\
\text { terminales. }\end{array}$ \\
\hline Certificación & $\begin{array}{c}\text { No originan } \\
\text { calificaciones. }\end{array}$ & $\begin{array}{l}\text { Pueden o no ser } \\
\text { calificadas. }\end{array}$ & Son calificables. \\
\hline $\begin{array}{l}\text { Procesos que } \\
\text { originan }\end{array}$ & Nivelación. & $\begin{array}{c}\text { Ayuda y refuerzo } \\
\text { para el alumno } \\
\text { Métodos alternativos } \\
\text { de aprendizaje si es } \\
\text { necesario. }\end{array}$ & $\begin{array}{l}\text { Refuerzo para el } \\
\text { alumno. } \\
\text { Reorientación de la } \\
\text { planificación si es } \\
\text { necesario. }\end{array}$ \\
\hline Certificación & $\begin{array}{l}\text { No originan } \\
\text { calificaciones. }\end{array}$ & $\begin{array}{l}\text { Pueden o no ser } \\
\text { calificadas. }\end{array}$ & $\begin{array}{l}\text { Acreditación de los } \\
\text { resultados: } \\
\text { - Calificación } \\
\text { - Promoción } \\
\text { - titulación }\end{array}$ \\
\hline $\begin{array}{l}\text { Análisis de } \\
\text { resultados }\end{array}$ & Referida a criterios. & Referida a criterios. & $\begin{array}{l}\text { Resultados globales } \\
\text { con relación a } \\
\text { criterios o normas. }\end{array}$ \\
\hline
\end{tabular}

Fuente: Adaptado de Castro et al. (2004). 
Bordas y Cabrera (2001) hablan además de la evaluación formadora, es decir una evaluación que arranque del mismo discente y que se fundamente en el autoaprendizaje, frente a la simple evaluación formativa o estímulo que es una respuesta a la iniciativa docente. Una evaluación que arranque del análisis de los propios aciertos y errores.

\subsection{Tipos de evaluación según los criterios de valor}

Dependiendo de los criterios de valor utilizados para la evaluación los expertos distinguen los tipos de evaluación que se recogen a continuación:

\section{La Evaluación Idiográfica}

Esta evaluación toma como referente al propio individuo, teniendo en cuenta las capacidades iniciales del alumno y la estimación de sus posibilidades de aprendizaje a lo largo de un determinado período (curso, módulo, etc.) en función de sus circunstancias Castro et al. (2004). Se valora el rendimiento final alcanzado pero sobre todo el esfuerzo por aprender y formarse a lo largo del proceso, las actitudes.

\section{Evaluación nomotética}

Se trata de una evaluación respecto a indicadores externos. Se puede distinguir entre la evaluación normativa y a la evaluación criterial.

\section{A) La Evaluación Normativa}

Establece una valoración del individuo midiendo sus resultados con respecto a los del grupo o a una norma. Se trata de un criterio externo de modo que un alumno con un nivel medio en el logro de los objetivos y competencias puede resultar evaluado negativamente si el nivel de los miembros del grupo es elevado, o positivamente en un grupo con un nivel medio bajo (Castro et al., 2004). Es por esto que no parece útil para apreciar el progreso de un alumno según sus propias capacidades a pesar de que permita en ocasiones corregir el "ruido" o desviación debida a factores externos. Esta evaluación está en línea de la prioridad tradicional en el pasado de comparar unos individuos con otros (McDonald et al., 2000).

\section{B) La Evaluación CRITERIAl}

En este caso se establece una evaluación del alumno en relación con criterios externos predeterminados y concretos. En primer lugar se establecen los objetivos y competencias a alcanzar para después valorar la actuación del alumno respecto los mismos, independientemente de la situación en que se encuentran sus compañeros, por lo que corrige el fallo anteriormente planteado en la evaluación normativa (Castro et al., 2004). Según recoge el profesor Molnar" "Para ayudar al alumno importa más enseñarle hasta donde lo han conducido sus esfuerzos en el proceso de apren-

1. Molnar, G. (Recopilación): "Evaluación educativa: Conceptos y Definiciones", disponible en: http://www.chasque.apc.org/gamolnar/evaluacion\%20educativa/homeevaluacion.html 
dizaje, que el lugar que ocupa con relación a sus compañeros o una escala". (De Landsheere, 1985). Además, permite elaborar un diagnóstico de las dificultades y valorar la adecuación de la estrategia.

\section{Consideraciones SObRe los PROCEDimientos de EVAluaCión}

Tal y como recoge Font (2003), siguiendo la clasificación propuesta por Bloom (1975) se categoriza los niveles de abstracción de los procesos de aprendizaje en orden creciente:

1. Conocimiento (recordar cifras, fechas, sucesos, etc.).

2. Comprensión (comprender información, interpretarla, trasladarla a contextos nuevos, etc.).

3. Aplicación (utilizar la información, usar teorías, resolver problemas, etc.).

4. Análisis (percibir parámetros de organización y significados ocultos, identificar componentes, elementos, organizar partes, etc.).

5. Síntesis (crear nuevas ideas, relacionar, deducir, extraer conclusiones, etc.).

6. Evaluación (comparar y discriminar ideas, evaluar teorías, tomar decisiones razonadas, etc.).

Estos aspectos deberán ser tenidos en cuenta en la elección de uno u otro procedimiento. Deberá analizarse además la finalidad, el objetivo, el rigor métrico y los costes que conlleva cada una de las opciones o instrumentos evaluativos ${ }^{2}$. A medida que aumente la complejidad de la tarea a ser evaluada, más difícil será la definición de los criterios para evaluar las competencias adquiridas (De Landsheere, 1985). Escudero (2010) ordena de más abiertos a más cerrados los siguientes procedimientos de evaluación:

- Observación y seguimiento.

- Procedimientos orales ${ }^{3}$.

- Ejercicios escritos. Temas y ensayos.

- Trabajos e informes.

- Ejercicios y ensayos cortos.

- Problemas y aplicaciones.

- Instrumentos cerrados.

De acuerdo con Font (2003) se debe evitar cualquier sistema de evaluación de acto único, sea cual sea su modalidad (examen oral, escrito, o de respuesta múlti-

2. Por ejemplo, la opción de elegir entre varias preguntas del examen puede generar en el alumno una percepción de lotería así como una disminución de la dificultad, ocasionando una reducción del esfuerzo por parte del alumno (Wainer y Thissen, 1994).

3. La utilización de entrevistas para la evaluación de los alumnos sólo es viable en situaciones donde no exista masificación, pero además debe tenerse en cuenta que en ocasiones los requerimientos burocráticos obligan a disponer de constancia escrita de la evaluación por lo que con la entrevista no se cumpliría esta obligación. 
ple), puesto que esto impide reflejar la complejidad asociada a los procesos de aprendizaje. Por ejemplo, los exámenes no ofrecen información sobre los niveles más altos de la taxonomía de Bloom (aplicación, análisis, síntesis, evaluación). Por el contrario, se deberían considerar todos los elementos y procesos relacionados con el objeto de evaluación. Además, la triangulación de procedimientos sirve de apoyo a la validez de la evaluación (Escudero, 2010).

A la hora de determinar los procedimientos de evaluación deben tener en cuenta en primer lugar las siguientes premisas (Font, 2003):

- El sistema de evaluación elegido condiciona el método de aprendizaje e influye en el aprendizaje mismo.

- La alternativa a un sistema de evaluación de acto único debe consistir en una metodología procesal.

- La evaluación implica un compromiso entre las partes que participan de un mismo proceso.

De forma más amplia, Escudero (1995) ofrece algunas estrategias de mejora que, con carácter general, resultan útiles para conseguir una evaluación de calidad y coherente:

i. Ajustar los procedimientos de la evaluación a los objetivos y destrezas que se quieren evaluar. En el diseño de las evaluaciones el profesor debe buscar la mayor correlación posible entre los procedimientos evaluativos utilizados y las competencias requeridas al estudiante.

ii. Diversificar los procedimientos de evaluación. Dado que los objetivos perseguidos en una materia suelen ser amplios, resultará adecuado utilizar procedimientos y aproximaciones evaluativas diversas, apropiadas para determinar el grado de logro de toda la variedad de competencias requeridas. Pero además, esta diversidad permitirá reducir el riesgo de error en la determinación de la calificación final otorgada al alumno.

iii. Establecer de modo claro y preciso y dar a conocer a los alumnos, desde el principio, los detalles relativos a los criterios que se van a utilizar en el proceso evaluativo (contenidos, plazos, etc.) para facilitarles la planificación su esfuerzo y sus estrategias de estudio.

iv. Ofrecer retroalimentación a los estudiantes, proporcionándoles información efectiva (feed-back) a lo largo de la interacción didáctica sobre los puntos fuertes y débiles en el aprendizaje y en las evaluaciones, que les sirva de elemento de estímulo para el aprendizaje.

v. Reflexionar críticamente y en profundidad sobre los resultados obtenidos en las evaluaciones, asumiendo nuestra parte de responsabilidad en los resultados para buscar oportunidades de mejora.

\section{Metodología}

El objetivo del trabajo es comprobar el grado de utilización de los diferentes tipos de evaluación anteriormente comentados, atendiendo a la opinión de primera mano 
expresada por los estudiantes, como receptores y principales protagonistas en general en el proceso de enseñanza-aprendizaje, y en particular en el proceso de evaluación. Dado el enfoque eminentemente aplicado que se desea ofrecer en el estudio se ha optado por utilizar una fuente de información primaria como es el cuestionario para el desarrollo del trabajo. Los alumnos son los principales usuarios de la enseñanza y por tanto su opinión resulta la fuente de información más directa. Si bien no está exento de limitaciones técnicas y de contraindicaciones, el cuestionario resulta muy válido y útil cuando se emplea debidamente (Escudero, 2000). Más si se tiene en cuenta que a pesar de la existencia de gran número de trabajos centrados en diferentes aspectos relacionados con los estudiantes de educación superior, en muchos de ellos los alumnos únicamente se consideran un elemento pasivo a estudiar, pero no sujetos activos que participan expresando sus opiniones y consideraciones (Pinelo, 2005).

En concreto se utilizó una muestra de alumnos pertenecientes a la modalidad semipresencial de la Licenciatura en Ciencias del Trabajo impartida en la Facultad de Ciencias Sociales y Humanas de la Universidad de Zaragoza, localizada en el Campus de Teruel en el curso 2007/2008. Dichos estudiantes combinan la educación a distancia apoyada en la utilización de las TIC con la educación tradicional presencial (Marzo et al., 2006).

El cuestionario se diseñó dentro de un proyecto más amplio de revisión y análisis de la opinión de los estudiantes respecto a temas relacionados con la evaluación tales como los procedimientos e instrumentos concretos de evaluación más utilizados. Dentro del mismo, partiendo de las dimensiones principales que se desean analizar en este trabajo, se incluyeron preguntas sobre el grado de utilización de los distintos tipos de evaluación de sus resultados en el sistema universitario actual al objeto de recabar la opinión de los alumnos al respecto.

Para ello se eligió una escala de tipo Likert de 1 a 5 que permitiera reflejar el grado de uso de los distintos tipos de evaluación en los siguientes términos: 5= Mucho, 4= Bastante, $3=$ Medio, $2=$ Poco y $1=$ Nada. En el Anexo I se recoge el detalle de las preguntas referentes a los tipos de evaluación analizados en el presente estudio.

Los cuestionarios se facilitaron personalmente a todos los alumnos que participaron en las sesiones presenciales a las que asisten varios sábados al mes. De este modo se pudo explicar de primera mano los objetivos de la presente investigación a los estudiantes. Adicionalmente se utilizó la página web de la titulación para poner a disposición de todos los estudiantes, tanto aquellos que asisten regularmente a las sesiones presenciales como aquellos que asisten de forma esporádica. Las instrucciones para cumplimentar el cuestionario estaban incluidas en el mismo, de modo que el alumno pudo revisarlas independientemente del lugar y momento de realización de la encuesta. En todo caso, los encuestados completaron las encuestas fuera del horario de clases presenciales para no interferir en el funcionamiento de las mismas y para conseguir el anonimato de los alumnos puesto que el número de estudiantes por clase es reducido y se desea evitar la presión derivada de la identificación del encuestado por parte del profesor. En todo caso, algunos de los alumnos remitieron el cuestionario, una vez completado, a través del correo electrónico con lo cual podría resultar fácil su identificación. No obstante, las preguntas no se refieren a una asignatura o profesor concreto 
sino a patrones generales o niveles medios por lo que el cuestionario no resulta excesivamente comprometedor, evitando que esto se convirtiese en un factor de disuasión a la hora de colaborar en el proyecto. Además, en todo momento se le asegura al alumno la confidencialidad en el tratamiento de sus respuestas. El número total de cuestionarios recibidos a través de las distintas vías fue de 46, todos ellos válidos, por lo que este es el tamaño final de la muestra.

\section{Resultados}

Como ya se ha indicado anteriormente, el presente trabajo se encuentra enmarcado dentro de un estudio más amplio sobre el estudio de la opinión de los estudiantes universitarios ante diferentes aspectos de la evaluación. En concreto, en este trabajo la atención se centra en la opinión referente a los tipos de evaluación más utilizados en la universidad. Del análisis de las informaciones ofrecidas por los estudiantes, se pueden extraer algunas luces y sombras que se comentan a lo largo del presente apartado. A continuación se recoge la revisión de los resultados relativos a los tipos de evaluación realizada por los profesores, distinguiendo entre la evaluación inicial, la evaluación estímulo y la evaluación sumativa por un lado, y la evaluación normativa y criterial, por otro.

\subsection{La evaluación inicial}

Como se ha explicado anteriormente, la evaluación inicial permite obtener un diagnóstico de la situación de partida del alumno, información que resulta muy valiosa y que debe ser analizada previamente al inicio del proceso. Esta variable se ha medido a través del análisis, por un lado del nivel de utilización de la "evaluación al inicio de la asignatura" y por otro del nivel de utilización de la "evaluación al inicio de cada uno de los temas". Según muestran los resultados, el grado de utilización de esta evaluación por parte del profesorado es intermedio, con una media de 2,54 sobre 5 . Sin embargo, si revisamos los datos concretos, tal y como se observa en la Tabla 1, son más de un 30\% los encuestados que califican como nulo el uso de este tipo de evaluación, bien sea al inicio de la materia o al inicio de cada uno de los bloques temáticos.

Tabla 1. Utilización de la evaluación inicial.

\begin{tabular}{|l|c|}
\hline Grado de uso & Porcentaje \\
\hline Nada & $30,43 \%$ \\
\hline Poco & $19,57 \%$ \\
\hline Medio & $26,09 \%$ \\
\hline Algo & $13,04 \%$ \\
\hline Mucho & $10,87 \%$ \\
\hline Total & $100 \%$ \\
\hline
\end{tabular}

Fuente: Elaboración propia. 
Estos resultados son coherentes con los obtenidos en el trabajo de Escudero (1995), por lo que se puede concluir que poco ha cambiado en más de diez años. A pesar de reconocer la importancia de un diagnóstico inicial mediante evaluaciones iniciales, gran parte de los profesores universitarios no realizan tal análisis.

Si diferenciamos entre la utilización de la evaluación al inicio de la materia y al inicio de cada uno de los temas los resultados son los siguientes:

Tabla 2. Detalle de la utilización de la evaluación inicial.

\begin{tabular}{|l|c|c|}
\hline \multirow{2}{*}{ Grado de uso } & Al inicio de la materia & Al inicio de los temas \\
\cline { 2 - 3 } & Porcentaje & Porcentaje \\
\hline Nada & $26,09 \%$ & $34,78 \%$ \\
\hline Poco & $26,09 \%$ & $13,04 \%$ \\
\hline Medio & $26,09 \%$ & $26,09 \%$ \\
\hline Algo & $8,70 \%$ & $17,39 \%$ \\
\hline Mucho & $13,04 \%$ & $8,70 \%$ \\
\hline Total & $100 \%$ & $100 \%$ \\
\hline
\end{tabular}

Fuente: Elaboración propia.

Se observan ligeras diferencias en los extremos de la tabla. Por un lado, parece que la ausencia de este tipo de evaluación es más habitual en lo referente a la evaluación al inicio de los temas que a la evaluación al inicio de la materia. Además, a penas el $8,7 \%$ de los estudiantes afirman que existe un alto grado de utilización de la evaluación al inicio de los temas, mientras que este valor alcanza el 13,04\% en lo referente a la evaluación al inicio de la materia. Esto es coherente con el sistema semipresencial en el que el contacto directo con el alumno coincide con el inicio de la asignatura, pero tras esa primera reunión el alumno puede decidir sobre el modo de seguimiento de la asignatura. No obstante, el contacto permanece abierto en todo momento a través de la web de la asignatura y las distintas herramientas que incorpora como el foro, chat o correo electrónico, a través de los cuales se puede gestionar la evaluación inicial al principio de cada uno de los temas o bloques temáticos.

Por otra parte, sigue observándose que tanto en la evaluación al inicio de los bloques temáticos como de la materia en su conjunto, el porcentaje de estudiantes que indican que no se lleva a cabo dicha evaluación, o escasamente, alcanza valores próximos al 50\% de los encuestados.

\subsection{La evaluación estímulo}

Respecto a la evaluación estímulo o evaluación formativa, se ha recogido en el cuestionario a través de la variable que recoge el grado de uso de "exámenes par- 
ciales" o de "preguntas en clase sobre la materia". En este caso, los datos son más optimistas, puesto que reflejan cómo gran parte del profesorado realiza un esfuerzo considerable por llevar a cabo un seguimiento y control permanente del aprendizaje a lo largo del proceso de enseñanza-aprendizaje de los alumnos. En concreto el valor medio en esta variable es de 3,43 sobre 5, y tal como se puede apreciar en la Tabla 3, más de la mitad de los estudiantes consideran importante el nivel de uso de este tipo de evaluación, tanto a través de exámenes parciales, como directamente y de modo menos formal, a través de preguntas directas en clase.

Tabla 3. Utilización de la evaluación estímulo.

\begin{tabular}{|l|c|}
\hline Grado de uso & Porcentaje \\
\hline Nada & $6,52 \%$ \\
\hline Poco & $15,22 \%$ \\
\hline Medio & $26,09 \%$ \\
\hline Algo & $26,09 \%$ \\
\hline Mucho & $26,09 \%$ \\
\hline Total & $100 \%$ \\
\hline
\end{tabular}

Fuente: Elaboración propia.

Al distinguir entre la utilización de exámenes parciales y la utilización de preguntas en clase como modo de obtener elementos para la evaluación del alumno se observa que resulta relativamente más habitual el uso de los exámenes parciales. A pesar del interés de la utilización de las preguntas en clase como modo de retroalimentación y de medición del nivel de la clase, puede resultar complicado articular un sistema de evaluación objetivo y equitativo para todos los estudiantes. La disponibilidad de tiempo en la clase es limitada por lo que trasladar tales observaciones, en ocasiones incompletas, como elementos objetivos en la evaluación de todos los alumnos puede resultar más complicado que en el caso de los exámenes parciales formalmente establecidos.

Tabla 4. Detalle de la utilización de la evaluación estímulo.

\begin{tabular}{|l|c|c|}
\hline \multirow{2}{*}{ Grado de uso } & Exámenes parciales & Preguntas en clase \\
\cline { 2 - 3 } & Porcentaje & Porcentaje \\
\hline Nada & $0,00 \%$ & $13,04 \%$ \\
\hline Meco & $13,04 \%$ & $17,39 \%$ \\
\hline Algo & $26,09 \%$ & $26,09 \%$ \\
\hline Mucho & $30,43 \%$ & $21,74 \%$ \\
\hline Total & $30,43 \%$ & $21,74 \%$ \\
\hline
\end{tabular}

Fuente: Elaboración propia. 
En todo caso, sigue observándose un alto uso de las preguntas en clase como elemento de evaluación estímulo, alcanzando casi el 70\% el porcentaje de alumnos que indican que se da una utilización media o superior de este tipo de evaluación estímulo. En resumen, parece haberse avanzado respecto a la situación descrita por Escudero (1995), donde sólo un 15\% del alumnado manifestaba que los profesores formulaban preguntas en clase sobre los aspectos vistos.

\subsection{La evaluación sumativa}

La utilización de la evaluación sumativa se recoge en el cuestionario a través de una variable que pregunta al encuestado sobre el grado de utilización de la "evaluación únicamente a través de éxamenes finales". El valor medio otorgado a la variable que recoge el uso global de la evaluación sumativa alcanza valores muy próximos a los de la evaluación estímulo un 3,22, incluso ligeramente superiores en el caso de la evaluación estímulo. Esto parece reflejar un desplazamiento de la importancia otorgada por el profesor a la evaluación sumativa hacia la evaluación formativa como parte del proceso. Frente a los resultados mostrados por Escudero (1995), donde el peso de los exámenes finales era abrumador, nuestros datos reflejan un mayor peso de la evaluación estímulo o formativa respecto a la evaluación sumativa, lo que refleja un incremento en la importancia otorgada a la evaluación como elemento con valor pedagógico.

Tabla 5. Utilización de la evaluación sumativa.

\begin{tabular}{|l|c|}
\hline Grado de uso & Porcentaje \\
\hline Nada & $13,04 \%$ \\
\hline Poco & $13,04 \%$ \\
\hline Medio & $30,43 \%$ \\
\hline Algo & $26,09 \%$ \\
\hline Mucho & $17,39 \%$ \\
\hline Total & $100 \%$ \\
\hline
\end{tabular}

Fuente: Elaboración propia.

Respecto a la evaluación nomotética, se han incluido en el cuestionario dos preguntas sobre la "evaluación del alumno con respecto a sus capacidades" y "evaluación del alumno con respecto al grupo", para recoger las variables referentes a la evaluación normativa y criterial, respectivamente. Los resultados presentan niveles medios tanto para la evaluación normativa, en función del nivel de los miembros del grupo como para el caso de la evaluación criterial. En ambos casos el mayor porcentaje de respuestas se encuentran en los términos medios de la tabla, por lo que puede concluirse que se utilizan de forma conjunta tanto evaluaciones normativas como criteriales. 
Tabla 6. Utilización de las evaluaciones normativa y criterial.

\begin{tabular}{|l|c|c|}
\hline \multirow{2}{*}{ Grado de uso } & Evaluación Normativa & Evaluación Criterial \\
\cline { 2 - 3 } & Porcentaje & Porcentaje \\
\hline Nada & $8,70 \%$ & $8,70 \%$ \\
\hline Poco & $13,04 \%$ & $21,74 \%$ \\
\hline Medio & $39,13 \%$ & $43,48 \%$ \\
\hline Algo & $30,43 \%$ & $13,04 \%$ \\
\hline Mucho & $8,70 \%$ & $13,04 \%$ \\
\hline Total & $100 \%$ & $100 \%$ \\
\hline
\end{tabular}

Fuente: Elaboración propia.

\section{Conclusiones}

Tras la revisión de los datos ofrecidos a lo largo del trabajo pueden extraerse diversas conclusiones. Por un lado puede decirse que, si bien se debe conceptualizar la evaluación como un proceso continuo y sistemático, las deficiencias del sistema tradicional de evaluación, han desvirtuado la propia naturaleza del mismo al conceder excesiva importancia al resultado y ocasionar que el alumno conciba el proceso formativo como un modo de alcanzar el citado resultado.

Además de su papel tradicional para conocer el rendimiento del alumno, la evaluación debe permitir diagnosticar el nivel o la situación en la que se encuentra el estudiante, ofrecerle retroinformación sobre sus puntos fuertes y débiles y motivarle para lograr los resultados esperados. El alumno debe recibir información referente al grado de logro de los distintos objetivos o competencias marcadas (feed-back) que le permita ajustar o adaptar sus esfuerzos. Para ello resulta importante que el profesor desarrolle tanto una evaluación inicial de diagnóstico, como posteriores evaluaciones formativas.

Los resultados obtenidos en un primer análisis exploratorio muestran todavía un escaso uso de la evaluación inicial o de diagnóstico para comprobar los conocimientos y las competencias que posee el alumno antes de iniciar el proceso formativo. No obstante, se observa un cierto avance hacia el uso de la evaluación formativa o estímulo que permite que la evaluación se lleve a cabo a lo largo del proceso de enseñanza-aprendizaje y que el alumno conozca sus avances sin esperar al final del mismo. Esto resulta importante puesto que esta evaluación permite adoptar medidas tanto por parte del alumno como del profesor cuando existen desviaciones, mientras que con el uso de una evaluación únicamente sumativa alumno y profesor conocen los resultados al final del proceso, cuando estos únicamente presentan una función punitiva.

En definitiva, la evaluación debe formar parte del proceso de enseñanza-aprendizaje con un carácter formativo y continuo, no limitado al momento del examen 
final como tradicionalmente se ha considerado, para lo cual se deben constituir la evaluación como eje vertebrador del proceso que ofrece oportunidades de mejora para facilitar y apoyar el logro de la excelencia en las instituciones universitarias.

\section{REFERENCIAS BIBLIOGRÁFICAS}

BANGERT, R.; KULIK, C.; KULIK, J. y MORGAN, M. (1991). The instructional effect of feedback in test-like events. Review of Educational Research, 61, 213-238.

BIGGS, J. (1996). Assessing Learning Quality: Reconciling institutional, staff and educational demands. Assessment and Evaluation in Higher Education, 21, 1, 5-15.

BIGGS, J. (2005). La calidad del aprendizaje universitario. Madrid: Narcea.

BLOOM, B.S. (1975). Taxonomía de los objetivos de la educación: la clasificación de las metas educacionales. Buenos Aires: El Ateneo.

BOL, L. y STRAGE, A. (1996). The contradiction between teachers' instructional goals and their assessment practices in high school biology courses. Science Education, 80, 145-163.

BORDAS, M. I. y CABRERA, F. A. (2001). Estrategias de evaluación de los aprendizajes centrados en el proceso. Revista Española de Pedagogía, 218, 25-48.

CAMPANARIO, J. M. (1998). Preguntas y respuestas sobre la evaluación de los alumnos en la enseñanza de las ciencias. Tarbiya: Revista de Investigación e Innovación Educativa, 19, 69-84.

CASTRO, F.; CORREA, M. E. y LIRA, H. (2004). Curriculum y evaluación. Texto Guía. Chillan: Universidad del Bío-Bío.

CÓRDOBA, F. J. (2006). La evaluación de los estudiantes: una discusión abierta. Revista Iberoamericana de Educación, 39. Disponible en: http://www.rieoei.org/ deloslectores/1388Cordoba-Maq.pdf

CROOKS, J. (1988). The Impact of Classroom Evaluation Practices on Students. The Review of Educational Research, 58 (4), 438-481.

DOCHY, F. (2001). A new assessment era: different needs, news challenges. Research Dialogue in Learning and Instruction, 2, 11-20.

ESCUDERO, T. (1995). Evaluación de los aprendizajes en la universidad. En Actas del Simposium de Innovación Universitaria "Diseño, desarrollo y evaluación del currículum universitario" (pp. 371-396). Barcelona: Servicio de Publicaciones Universitat Autònoma de Barcelona.

ESCUDERO, T. (2000). La voz de los estudiantes: un delicado instrumento de evaluación. Cuadernos IRC, 5, 31-38.

ESCUDERO, T. (2010). Sin tópicos ni malentendidos: fundamentos y pautas para una práctica evaluadora de calidad en la enseñanza universitaria. Colección Documentos Instituto de Ciencias de la Educación, Documento 09. Zaragoza: Universidad de Zaragoza.

FONT, A. (2003). Una experiencia de autoevaluación y evaluación negociada en un contexto de aprendizaje basado en problemas (ABP). Revista de la Red Estatal de Docencia Universitaria, 3 (2), 100-112. 
DE LANDSHEERE, G. (1985). Diccionario de la evaluación y de la investigación educativa. Barcelona: Oikos-Tau.

MARZO, M.; ESTEBAN, L. y GARGALLO, A. (2006) ¿Inciden las nuevas tecnologías en los resultados alcanzados por los alumnos? Un estudio exploratorio. Revista de Educación, Ministerio de Educación y Ciencia, 340, 695-711.

MCDONALD, R.; BOUD, D.; FRANCIS, J. y GONCZI, A. (2000). Nuevas perspectivas sobre la evaluación. Boletín Cinterfor: Boletín Técnico Interamericano de Formación Profesional, 149, 41-72.

PÉREZ, M. L.; CARRETERO, R.; PLAMA, M. y RAFEL, E. (2000). La evaluación de la calidad del aprendizaje en la Universidad. Infancia y Aprendizaje, 91, 5-30.

PINELO, F.T. (2005). La Evaluación en el Proceso Enseñanza-Aprendizaje desde la Perspectiva del Alumno. El Caso de la Carrera de Psicología en la FES Zaragoza/UNAM. Revista Mexicana de Orientación Educativa, 6. Disponible en: http://www.remo.ws/revista/n6/n6-pinelo.htm.

ROSALES, C. (1981). Criterios para una evaluación formativa. Madrid: Narcea.

ROSALES, C. (2000). Evaluar es reflexionar sobre la enseñanza. Madrid: Narcea.

SCRIVEN, M. (1967). The methodology of evaluation. En TYLER, R. W.; GAGNÉ, R. M. y SCRIVEN, M. (Eds.). Perspectives of curriculum evaluation (pp. 39-83). Chicago: Rand McNally.

WAINER, H. y THISSEN, D. (1994). On examinee choice in educational testing. Review of Educational Research, 6(1), 159-195. 


\section{Anexo 1. Preguntas de cuestionario referentes a la utilización de DISTINTOS TIPOS DE EVALUACIÓN}

Sexo: $\square$ Hombre / $\square$ Mujer. Curso-Titulación-Universidad:

* Valore el grado de utilización de las distintas evaluaciones, procedimientos y metodologías para determinar los conocimientos previos, intermedios y finales del alumno ( $1=$ Nada $5=$ Mucho).

\begin{tabular}{|l|c|c|c|c|c|}
\hline Tipo de evaluación: & 1 & 2 & 3 & 4 & 5 \\
\hline Evaluación al inicio de la asignatura & 1 & 2 & 3 & 4 & 5 \\
\hline Evaluación al inicio de cada uno de los temas & 1 & 2 & 3 & 4 & 5 \\
\hline Exámenes parciales & 1 & 2 & 3 & 4 & 5 \\
\hline Preguntas en clase sobre la materia & 1 & 2 & 3 & 4 & 5 \\
\hline Evaluación a través de examen final únicamente & 1 & 2 & 3 & 4 & 5 \\
\hline Evaluación del alumno con respecto sus capacidades & 1 & 2 & 3 & 4 & 5 \\
\hline Evaluación del alumno con respecto al grupo & 1 & 2 & 3 & 4 & 5 \\
\hline Evaluación del alumno con respecto a un nivel fijo & & &
\end{tabular}

\section{$[\ldots]$}

Muchas gracias por su colaboración, le garantizamos la confidencialidad de sus respuestas. No se dará publicidad ni se ofrecerá información a terceros de los cuestionarios individuales. 